\title{
Relación entre el déficit sensorial auditivo y depresión en personas mayores: revisión de la literatura
}

\author{
José Carlos Millán-Calenti ${ }^{\mathrm{a}}$, , Ana Maseda ${ }^{\mathrm{a}}$, Sophie Rochette ${ }^{\mathrm{a}}$ e Isabel García- \\ Monasterio $^{\text {b }}$ \\ ${ }^{a}$ Grupo de Investigación en Gerontología, Universidad de A Coruña, A Coruña, España \\ ${ }^{b}$ Complejo Hospitalario Universitario Juan Canalejo-Marítimo de Oza, A Coruña, España
}

\section{Resumen}

Los avances en las condiciones sanitarias y socioeconómicas de los países desarrollados han incrementado la esperanza de vida y el número de personas mayores. Sin embargo, aunque las condiciones sanitarias han mejorado, las alteraciones de la salud en relación con la edad siguen aumentando, siendo el déficit sensorial auditivo una de las más frecuentes. De origen multifactorial, en donde los cambios morfofuncionales en relación con el envejecimiento van a desempeñar su papel, presentará predisposiciones subyacentes en cada individuo.

El déficit sensorial auditivo va a influir negativamente sobre la calidad de vida de las personas mayores debido a las interferencias producidas sobre la capacidad para comunicarse, afectando, además, al estado de ánimo y al nivel de participación social, independientemente del estado cognitivo y físico del individuo, lo que a largo plazo, y en muchos casos, desembocará en un trastorno depresivo.

Detectar y tratar precozmente dicho déficit generará un importante beneficio bio-psico-social y funcional a la persona.

Palabras clave: Déficit sensorial auditivo, Presbiacusia, Depresión, Comunicación, Participación social

\section{Relationship between sensory hearing loss and depression in elderly people: a literature review}

\begin{abstract}
Advances in health, social and economic conditions in the developed countries have increased life expectancy and the number of elderly people. However, although health conditions have improved, age-related diseases are still increasing. One of the most common ailments is the age-related hearing loss, which has several pathophysiological causes and may be influenced by age-related morpho-functional changes. Hearing loss may also have underlying conditions in each individual.

Sensory hearing loss tends to negatively affect the quality of life of the elderly, interfering with their capacity to communicate and affecting mood and the level of participation in social life. This may be independent of the cognitive and physical state of individuals, which in the long term and in many cases may end in depression. Detection and early treatment of hearing loss is an important bio-psycho-social benefit to the elderly.
\end{abstract}

Keywords: Sensory hearing loss, Presbycusis, Depression, Communication, Social interaction

\section{Introducción}

En términos generales, aún admitiendo una diferencia importante según el nivel de desarrollo de los distintos países, se está produciendo un envejecimiento progresivo de la población, que se hace más evidente en algunos países europeos, entre los que se encuentra España ${ }^{1}$. Se prevé que la población de personas mayores en todo el mundo aumente en unos 694 millones entre 1970 y 2025, o lo que es lo mismo en un 223\%, por lo que en 2025 se alcanzarán casi los 1.200 millones de personas con más de 60 años ${ }^{1}$.

El aumento en el número de personas mayores, junto con el incremento de la esperanza de vida, conllevan una mayor prevalencia de las denominadas como enfermedades edad-dependientes, en las que el propio envejecimiento morfofuncional actuará como factor predisponente, siendo el déficit sensorial auditivo una de ellas ${ }^{2}$. La falta de homogeneidad en los diferentes estudios imposibilita tener un registro 
global sobre la incidencia y la prevalencia de la pérdida de audición. En todo caso, se ha mostrado una prevalencia muy variable, entre el 30 y el 70\%2. En los Estados Unidos, un tercio de las personas mayores entre 65 y 75 años, y hasta la mitad cuando nos referimos a los mayores de 75 años, muestran una pérdida de audición ${ }^{3}$. En España, más de 825.000 personas presentan pérdida de capacidad auditiva, de los que aproximadamente el $70 \%$ son mayores de 65 años ${ }^{4}$.

Dentro del déficit auditivo en las personas mayores, se establecen una serie de modificaciones morfofuncionales propias del proceso del envejecimiento, que actúan como coadyuvantes de dicha alteración y que se engloban bajo la denominación de pérdida de audición relacionada con la edad o presbiacusia, y no es posible aislar dichas modificaciones de otros factores predisponentes, debiéndose de pensar entonces en una etiología multifactorial en la que intervendrán otros factores como los traumas acústicos, patologías crónicas (insuficiencia renal, diabetes, hipoavitaminosis A, etc.), consumo de determinados fármacos, así como diferencias interindividuales en genes de susceptibilidad ${ }^{2,5}$.

A diferencia de otras hipoacusias, como en las enfermedades autoimmunitarias, la enfermedad de Ménière, el neuroma acústico, la fístula perilinfática o la pérdida traumática por ruidos que pueden ser unilaterales $^{3}$, la presbiacusia cursa de manera simétrica, afectando, por tanto, a ambos oídos.

Considerada como un problema mayor de salud pública ${ }^{6}$, por su repercusión sobre el estado de bienestar emocional, físico y social del individuo ${ }^{7}$, en su progresión se producirá una pérdida en la percepción de los sonidos de frecuencias altas, modificándose el umbral para las bajas. Su consecuencia más inmediata es que va a interferir en el entendimiento del habla, en el procesamiento de las informaciones acústicas y en la localización de las fuentes de los sonidos, de manera más evidente en locales ruidosos ${ }^{5}$. Esta característica de la presbiacusia reflejada en la frase «puedo oírte, pero no puedo entender lo que dices» ${ }^{8}$ hace que se incluya dentro de las hipoacusias neurosensoriales ${ }^{9}$. La presencia del fenómeno conocido como «regresión fonémica», caída en los tonos agudos en la audiometría tonal acompañada de una audiometría vocal desproporcionadamente mala, es fundamental para poder establecer el diagnóstico de presbiacusia? ${ }^{9}$.

Un problema añadido es que, dada su evolución lenta y progresiva, la mayoría de las veces se acude tarde al especialista, pasando en ocasiones varios años desde la aparición del problema hasta su tratamiento.

Al no existir un tratamiento curativo, las medidas de intervención deberán de ir dirigidas a la prevención, mediante el adecuado control y modificación de los factores de riesgo, entre los que se incluyen la hipertensión arterial, la hipercolesterolemia y el hábito tabáquico, evitando las exposiciones a ruidos ambientales, especialmente cuando éstos son fuertes ${ }^{7}$. En muchas ocasiones resulta efectiva la utilización de audífonos o dispositivos electrónicos que amplifican y cambian el sonido para una mejor comunicación $^{10}$. Sin embargo, por diferentes razones, su utilización está poco extendida ${ }^{11}$, estableciéndose en algunos estudios en solamente una de cada 5 personas mayores de 65 años con problemas de audición ${ }^{12}$. En presbiacusias severas y precoces que no han podido beneficiarse del uso de audífonos, los implantes cocleares han proporcionado buenos resultados ${ }^{10}$. En otros casos, los fármacos vasodilatadores, las vitaminas del grupo $\mathrm{B}^{13}$, los antioxidantes y la restricción dietética ${ }^{14}$ han logrado algunos efectos, pero su eficacia está por demostrar.

Por otro lado, la depresión es uno de los principales trastornos mentales en las personas mayores. La prevalencia media de depresión mayor se sitúa alrededor del 1,8\%, mientras que la prevalencia media de síntomas depresivos clínicamente significativos sin llegar a cumplir los criterios de depresión mayor alcanza el $13,5 \%{ }^{15}$. No obstante, estos datos varían mucho en función del tipo de población estudiada, llegando incluso a duplicarse la tasa en pacientes mayores institucionalizados ${ }^{15}$. En todo caso, existe una alta proporción de casos de depresión no diagnosticada, por ser en las personas mayores un síndrome de especial complejidad debido a los cambios, tanto orgánicos como psicológicos y sociales, que tienen lugar con el envejecimiento ${ }^{7}$. Asimismo, se ha observado menos interés por los síntomas depresivos en la vejez debido a la creencia errónea de que en esta población constituyen una respuesta normal a la edad ${ }^{16}$.

Entre los principales factores que pueden influir en la expresión de la depresión, existen estudios ${ }^{17,18}$ que proponen algunas posibilidades de asociación con marcadores genéticos, a pesar de que no existen evidencias contundentes ${ }^{17}$, y la comorbilidad con otras enfermedades médicas es otro de los factores que ha recibido mayor atención. Las personas mayores con algún trastorno neurológico tienen una alta prevalencia de depresión $^{19}$. Así, es muy frecuente que en personas diagnosticadas de demencia aparezcan síntomas depresivos que suelen preceder al deterioro cognitivo ${ }^{20}$. Además, los trastornos cerebrovasculares pueden también predisponer, precipitar o perpetuar síntomas depresivos en la vejez, dando lugar a la denominada depresión vascular ${ }^{21}$. Se ha encontrado comorbilidad con otros trastornos neurológicos, como la enfermedad de Parkinson ${ }^{22}$, la esclerosis múltiple ${ }^{23}$ o la epilepsia ${ }^{24}$. Otros factores que pueden influir en la expresión de la depresión en la población de mayores con enfermedades neurológicas son los cambios anatómicos y fisiológicos en las áreas cerebrales y en los sistemas sensoriales, como la pérdida auditiva o visual ${ }^{25-27}$. Existe un síndrome denominado depresión de 
comienzo tardío que se utiliza para referirse a un subconjunto de pacientes con mayor cantidad de déficits neurológicos ${ }^{28}$ y que, en comparación con los mayores que desarrollan depresión a edades más tempranas, suelen tener menos antecedentes familiares de trastornos del estado de ánimo, una mayor prevalencia de demencia, peor ejecución en los tests neuropsicológicos, mayor deterioro auditivo neurosensorial, dilatación de los ventrículos laterales y más hiperintensidades en la sustancia blanca ${ }^{29}$.

Otras condiciones médicas pueden dar lugar a síntomas depresivos así como, diversos trastornos endocrinos, infecciones virales, infarto de miocardio, trastornos metabólicos o cáncer ${ }^{29}$. También se ha encontrado que la depresión puede sobrevenir frecuentemente en personas que presentan diabetes ${ }^{30}$, rotura de cadera $^{31}$ y artritis $^{32}$, lo que coincide con la teoría de que uno de los principales factores que pueden precipitar la aparición de un síndrome depresivo en la población anciana es el bajo estado funcional $^{33}$ secundario a alguna enfermedad física o neurológica ${ }^{34}$.

En relación con los factores psicológicos que pueden precipitar o predisponer a la aparición de síntomas de depresión, estarían los rasgos de personalidad, las distorsiones cognitivas y el control emocional $^{34,35}$. Existen investigaciones ${ }^{36}$ en las que se asume que los rasgos de personalidad son un factor de vulnerabilidad a la depresión en la vejez. Las características de personalidad que más se han estudiado han sido el neuroticismo, la maestría y la auto-eficacia ${ }^{37}$. Así, se encontró que una puntuación alta en neuroticismo y baja en maestría y en auto-eficacia eran buenos predictores del comienzo de síntomas depresivos en una población de mayores no institucionalizados ${ }^{38}$. También se encontró que el neuroticismo predecía la persistencia de la depresión en pacientes ambulatorios ${ }^{39}$. Además de los efectos directos que los rasgos de personalidad pueden tener sobre los síntomas depresivos, también interactúan con otros factores; así, por ejemplo, la interpretación que las personas hacemos acerca de los acontecimientos estresantes puede variar en función de nuestras características de personalidad y, por tanto, afectar también de manera indirecta a la manifestación de los síntomas ${ }^{40}$.

En cuanto a los precipitantes de tipo social, los sucesos vitales estresantes, el sufrimiento por la pérdida de una persona querida, el estrés crónico y los recursos sociales son factores importantes ${ }^{34}$. Una gran cantidad de trabajos revelan que el apoyo social está muy asociado con el desarrollo de depresión y además puede contribuir al impacto que las enfermedades crónicas tienen sobre la misma ${ }^{41}$. Los recursos psicosociales pueden influir sobre los síntomas depresivos de dos maneras: directamente, cuando estos recursos tienen un efecto beneficioso sobre los síntomas de depresión, independientemente de la presencia de un estresor, o a través de un efecto amortiguador, modificando la asociación entre los síntomas depresivos y el estresor. No obstante, la relación más estable y contundente entre los recursos sociales y los síntomas depresivos se ha encontrado en el apoyo percibido ${ }^{42}$.

En definitiva, la depresión en la vejez es un síndrome multifactorial y muy complejo que requiere tener en cuenta una diversidad de factores biológicos, psicológicos y sociales para poder realizar un diagnóstico correcto y un tratamiento eficaz.

Al realizar esta revisión, nuestro objetivo fue analizar la relación existente entre el déficit sensorial auditivo y la depresión en las personas mayores, al considerarla como uno de los elementos fundamentales en su pérdida de calidad de vida por su estrecha relación con una baja participación social.

\section{Material y métodos}

Se realizó una búsqueda bibliográfica de los estudios españoles y extranjeros que ofrecieran información específica y útil sobre aspectos relacionados con el déficit sensorial auditivo en personas mayores, los trastornos depresivos y la relación existente entre ambas patologías.

Para incluirlos en esta revisión, se consideraron los trabajos que cumplieran los siguientes criterios:

- Que incluyeran la población de interés, personas mayores.

- Que ofrecieran información sobre la prevalencia del déficit auditivo sensorial y de los trastornos depresivos en la población de personas mayores.

- Que analizaran la relación existente entre ambas patologías.

- Que definieran el déficit auditivo sensorial y los trastornos depresivos.

- Que incluyeran líneas de investigación que estudian la etiología y los diferentes factores de riesgo que influyen en la aparición del déficit auditivo sensorial y los trastornos depresivos.

- Que informasen de los métodos de detección, tratamientos preventivos y/o dispositivos de apoyo de los que pueden beneficiarse las personas mayores con pérdida auditiva.

- Que comentasen los factores biológicos, psicológicos y sociales asociados con déficit sensorial auditivo y/o trastorno depresivo.

- Que se refirieran a la comorbilidad de los trastornos depresivos con otras enfermedades médicas. 
La estrategia de búsqueda en la literatura científica incluyó varios procedimientos complementarios. Por un lado, se realizó una búsqueda en varias bases de datos bibliográficas (Medline y Web of Science) para referencias en español y en inglés, utilizando como límites temporales el período 1950-2010, pero con especial interés en los estudios más recientes, empleando las siguientes entradas: presbycusis, presbyacusis, age-related hearing loss, hearing loss, hearing impairment, combinadas con aging, ageing, elderly, old people, older adults, elderly and depression, depressive symptoms, depressed mood. Por otro lado, se llevó a cabo una búsqueda retrospectiva de las listas de referencias citadas en la bibliografía de los artículos encontrados y en las revisiones previas publicadas que se relacionaran con el tema tratado. Se revisaron además los índices de algunas de las revistas de la especialidad (Revista Española de Geriatría y Gerontología, Atención Primaria, Hearing Research, Gerontology, The Journals of Gerontology, Journal of the American Geriatrics Society).

\section{Déficit sensorial auditivo y depresión}

Es indiscutible que la pérdida de audición interfiere en la comunicación entre las personas y dificulta la interrelación del individuo con el medio en el que se desarrolla, dando lugar a un escaso ajuste social ${ }^{43}$. Además de que la pérdida de audición generará una especial dependencia de los cuidadores ${ }^{44}$; problemas a la hora de establecer estrategias de respuesta ante, por ejemplo, alarmas de emergencias, sentimientos de soledad, depresión y disminución de la calidad de vida, serían otras consecuencias a tener en cuenta ${ }^{45}$.

Diferentes estudios epidemiológicos ${ }^{46}$ relacionan la pérdida de audición y la depresión (tabla 1), determinándose un porcentaje mayor de depresión entre los pacientes mayores ingresados con déficit auditivo bilateral que entre los sujetos que oyen bien ${ }^{47,48}$. Además, los pacientes mayores con depresión de comienzo tardío y sin deterioro cognitivo presentaban más déficits auditivos que los pacientes con depresión de comienzo más temprano ${ }^{27}$. En un estudio ${ }^{49}$ realizado con personas mayores institucionalizadas, se encontró que aquéllos que presentaban dificultades auditivas, tenían más posibilidades de experimentar peor humor, asociando la severidad de la dificultad auditiva a altos niveles de síntomas depresivos y anhedonia.

Se ha observado una relación lineal entre la pérdida de la capacidad auditiva y el grado de depresión, de manera que a mayor déficit auditivo, mayor grado de depresión ${ }^{50}$. Otros estudios ${ }^{47}$ constatan que la utilización de dispositivos de apoyo auditivo disminuye e incluso previene la depresión en las personas mayores que tienen déficit auditivo, mejorando de este modo su calidad de vida. También se ha visto que personas mayores con implantes cocleares se benefician en aspectos como la comprensión del discurso e

informan de mejoras en los sentimientos de soledad y depresión ${ }^{51}$. No obstante, el beneficio del apoyo auditivo sobre la depresión no ha sido observado después de un año de seguimiento, y tampoco entre usuarios con prótesis auditivas de larga duración y los que la utilizaban por primera vez ${ }^{52}$. El tipo de aparato audio-tecnológico utilizado ${ }^{10}$ o la personalidad del usuario ${ }^{53}$ podrían explicar las diferencias observadas. En un trabajo ${ }^{54}$ realizado para determinar la interrelación entre la pérdida de audición, el apoyo social, la depresión y la ansiedad, se encontró que la pérdida de audición está relacionada moderadamente con la depresión y la ansiedad en aquellas personas con escaso apoyo social.

A pesar de su impacto sobre el nivel cognitivo de las personas mayores, la pérdida de audición parece afectar también a la función física ${ }^{55}$. Asociada de manera compleja a la depresión, la pérdida de audición puede afectar al estado funcional de la gente mayor, sin que exista un orden causal específico en su desarrollo ${ }^{45}$

\section{Conclusiones}

Aunque ambos procesos, déficit auditivo en personas mayores y depresión, presentan una alta prevalencia, la falta de homogeneidad en los estudios realizados dan unas cifras desiguales, por lo que sería interesante unificar criterios en las técnicas de cribado utilizadas a la vez que instaurar programas de prevención de salud, en donde se realicen revisiones protocolizadas y periódicas de audición, a fin de detectar y tratar precozmente este problema, lo que sin duda redundaría en corregir o retrasar sus complicaciones.

Por otra parte, seguir investigando en esta línea podría ayudar a establecer en qué medida el déficit sensorial auditivo en las personas mayores contribuye a aumentar el grado de aislamiento y, por consiguiente, de vulnerabilidad para desarrollar trastornos depresivos, además de facilitar la puesta en marcha de planes y programas de intervención que redujesen al máximo las consecuencias negativas para la vida y la salud contribuyendo a un envejecimiento saludable de la población. 
Tabla 1

Comparación de los aspectos metodológicos más relevantes de los principales estudios consultados relacionando el déficit sensorial auditivo y la depresión

\begin{tabular}{|c|c|c|c|c|c|}
\hline & $\mathrm{n}$ & Edad (años) & Estudio & Instrumentos valoración & $\begin{array}{l}\text { Resultados } \\
\text { principales/conclusiones }\end{array}$ \\
\hline Jones et ${ }^{46}$ & 657 & $\geq 70$ & $\begin{array}{l}\text { Transversal/entr } \\
\text { evistas }\end{array}$ & $\begin{array}{l}\text { - Audición: pregunta } \\
\text { «¿Tiene dificultad para oír } \\
\text { una conversación normal } \\
\text { (incluso con audífonos)?.» } \\
\text { No/poca/mucha } \\
\text { - Depresión: cuestionario } \\
\text { propio }\end{array}$ & $\begin{array}{l}\text { La prevalencia de la depresión es } \\
\text { mayor cuanto mayor es la } \\
\text { dificultad auditiva }\end{array}$ \\
\hline Abrams et al ${ }^{47}$ & 493 & $\begin{array}{l}\text { Media } \\
(74,3 \pm 5,3)\end{array}$ & $\begin{array}{l}\text { Transversal } \\
\text { prospectivo }\end{array}$ & $\begin{array}{l}\text { - Audición y depresión: } \\
\text { Clasificación Internacional } \\
\text { de Enfermedades, 9.a } \\
\text { revisión }\end{array}$ & $\begin{array}{l}\text { La tasa de depresión es mayor en } \\
\text { mayores con déficit auditivo que } \\
\text { en aquéllos que oyen bien }\end{array}$ \\
\hline Saito et $\mathrm{al}^{48}$ & 580 & $\geq 65$ & $\begin{array}{l}\text { Longitudinal, de } \\
\text { cohorte }\end{array}$ & $\begin{array}{l}\text { - Audición: HHIE } \\
\text { (Hearing Handicap } \\
\text { Inventory for the Elderly o } \\
\text { Inventario de discapacidad } \\
\text { auditiva para adultos } \\
\text { mayores) } \\
\text { - Depresión: GDS15 } \\
\text { (Escala dedepresión } \\
\text { geriátrica de } 15 \text { ítems) }\end{array}$ & $\begin{array}{l}\text { El déficit auditivo puede predecir } \\
\text { futuros síndromes depresivos }\end{array}$ \\
\hline Kalayam et $\mathrm{al}^{27}$ & 43 & $\geq 60$ & $\begin{array}{l}\text { Transversal, por } \\
\text { casos } \\
\text { consecutivos }\end{array}$ & $\begin{array}{l}\text { - Depresión: DSM-III-R } \\
\text { (Manual diagnóstico y } \\
\text { estadístico de los trastornos } \\
\text { mentales, 3.a ed. revisada) } \\
\text { y la HDRS (Escala de } \\
\text { valoración de Hamilton } \\
\text { para la evaluación de la } \\
\text { depresión) }\end{array}$ & $\begin{array}{l}\text { El diagnóstico y tratamiento de } \\
\text { déficit sensorial auditivo } \\
\text { desciende la morbilidad de } \\
\text { depresión de comienzo tardío. Se } \\
\text { observa mayor déficit auditivo en } \\
\text { pacientes con depresión de } \\
\text { comienzo tardío que en la de } \\
\text { comienzo temprano }\end{array}$ \\
\hline Brink \& Stones ${ }^{49}$ & 12.254 & $\geq 65$ & $\begin{array}{l}\text { Transversal por } \\
\text { casos } \\
\text { consecutivos }\end{array}$ & $\begin{array}{l}\text { - Audición y depresión: } \\
\text { MDS } 2.0 \text { (cuestionario del } \\
\text { conjunto mínimo de datos), } \\
\text { referido al deterioro } \\
\text { funcional auditivo (FHI) } \\
\text { con un solo ítem y a la } \\
\text { escala de depresión MSD } \\
2.0\end{array}$ & $\begin{array}{l}\text { El deterioro auditivo se asocia } \\
\text { con peor humor y éste con } \\
\text { síntomas depresivos y anhedonia. } \\
\text { Reducir los efectos negativos del } \\
\text { déficit auditivo incrementa la } \\
\text { calidad de vida }\end{array}$ \\
\hline \multirow[t]{2}{*}{ Cacciatore et $\mathrm{al}^{50}$} & 1.750 & $\begin{array}{l}\text { Media } \\
80,7 \pm 7,5) \geq \\
65\end{array}$ & Transversal & $\begin{array}{l}\text { - Audición: pregunta sobre } \\
\text { la capacidad de oír a otros } \\
\text { hablando en un tono } \\
\text { normal }(<28 \mathrm{~dB})\end{array}$ & $\begin{array}{l}\text { El déficit auditivo es muy común } \\
\text { en las personas mayores y se } \\
\text { asocia con el deterioro cognitivo, } \\
\text { la pérdida de capacidad funcional } \\
\text { y/o la depresión }\end{array}$ \\
\hline & & $\begin{array}{l}\text { Media } \\
(74,2 \pm 6,4)\end{array}$ & & $\begin{array}{l}\text { - Depresión: GDS (Escala } \\
\text { de depresión geriátrica) }\end{array}$ & \\
\hline
\end{tabular}


Tabla 1 (continuación)

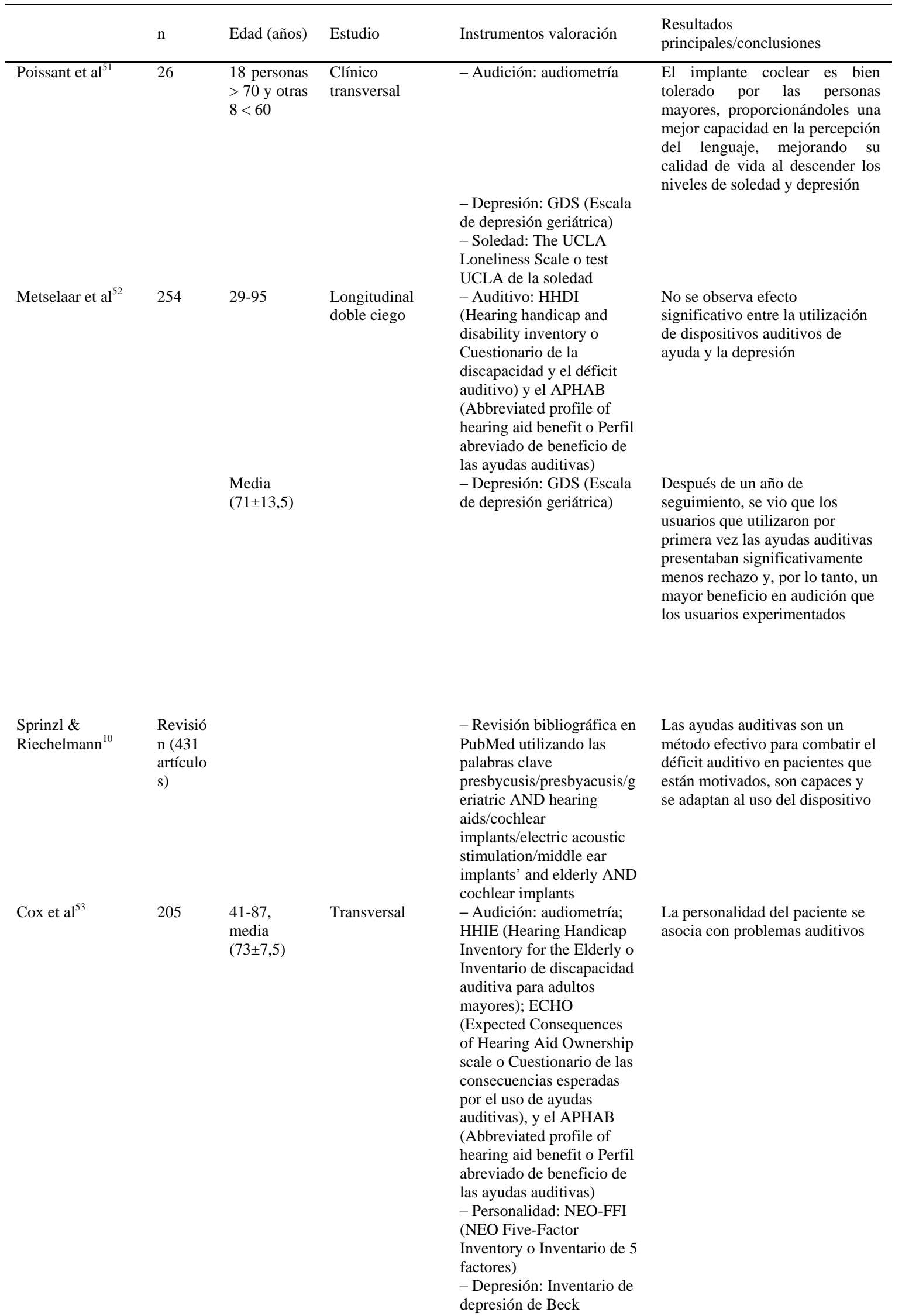


Tabla 1 (continuación)

\begin{tabular}{|c|c|c|c|c|c|}
\hline & $\mathrm{n}$ & $\begin{array}{l}\text { Edad } \\
\text { (años) }\end{array}$ & Estudio & Instrumentos valoración & $\begin{array}{l}\text { Resultados } \\
\text { principales/conclusiones }\end{array}$ \\
\hline Oppergard et $\mathrm{al}^{54}$ & 102 & $60-92$ & Transversal/entrevista & $\begin{array}{l}\text { - Audición: pregunta } \\
\text { directa sobre la calidad de } \\
\text { su audición (incluso con } \\
\text { dispositivos de ayuda) en } \\
\text { comparación a la mayoría } \\
\text { de gente de su edad: } \\
\text { Mejor que el resto/peor } \\
\text { que el resto }\end{array}$ & $\begin{array}{l}\text { La pérdida de audición está } \\
\text { relacionada con la depresión y } \\
\text { la ansiedad en personas } \\
\text { mayores, pero sólo en aquellos } \\
\text { casos con un menor acceso al } \\
\text { apoyo social y al apoyo de } \\
\text { cuidadores en una red social }\end{array}$ \\
\hline $\begin{array}{l}\text { López-Torres } \\
\text { Hidalgo et } \mathrm{al}^{55}\end{array}$ & 1.387 & $\geq 65$ & Transversal & $\begin{array}{l}\text { - Audición: HHIE-S } \\
\text { (Hearing Handicap } \\
\text { Inventory for the Elderly- } \\
\text { Short version o Versión } \\
\text { abreviada del Inventario } \\
\text { de discapacidad auditiva } \\
\text { para adultos mayores) } \\
\text { - Capacidad funcional: } \\
\text { Índice de Katz } \\
\text { - Depresión: GDS (Escala } \\
\text { de depresión geriátrica) }\end{array}$ & $\begin{array}{l}\text { Hay una alta prevalencia de } \\
\text { déficit auditivo asociado con } \\
\text { otras limitaciones funcionales }\end{array}$ \\
\hline Hogan et $\mathrm{al}^{45}$ & 43.233 & $\geq 55$ & Epidemiológico & $\begin{array}{l}\text { - Auditivo: SDAC } \\
\text { (Survey of Disability, } \\
\text { Ageing and Carers o } \\
\text { Encuesta sobre } \\
\text { discapacidad, } \\
\text { envejecimiento y } \\
\text { cuidadores) } \\
\text { - Calidad de vida: SF-12 } \\
\text { (Short Form of } 12 \text { Health } \\
\text { Survey o Cuestionario } \\
\text { abreviado de Salud SF- } \\
\text { 12) }\end{array}$ & $\begin{array}{l}\text { La discapacidad auditiva se } \\
\text { relaciona con la calidad de vida. } \\
\text { Las personas con déficit } \\
\text { auditivo - incluidas aquellas } \\
\text { que utilizan ayudas auditivas y } \\
\text { no presentan limitaciones en la } \\
\text { comunicación - tienen un peor } \\
\text { estado de salud mental y física }\end{array}$ \\
\hline
\end{tabular}

Paralelamente, es importante estudiar este problema desde la perspectiva social, valorar la tendencia a la soledad que tienen las personas mayores, observar sus relaciones interpersonales y aportarles desde la sociedad la atención y las ayudas necesarias para que no se sientan excluidos.

Establecer una relación biunívoca entre la deprivación sensorial auditiva bilateral y la depresión en la vejez resulta difícil debido a que, en esta población, la depresión puede ser consecuencia de numerosos factores, tanto físicos como psicosociales.

Entendemos que existen evidencias demostradas de la relación entre el déficit sensorial auditivo y la expresión de la depresión en las personas mayores, por lo que cualquier medida preventiva o de intervención sobre el primer elemento contribuirá a disminuir la prevalencia del segundo y de manera directa a incrementar el estado de bienestar de la población de personas mayores.

\section{Declaración de conflicto de intereses}

Los autores declaran no tener ningún conflicto de intereses.

\section{Bibliografía}

1. Organización Mundial de la Salud. Envejecimiento activo: un marco político. Rev Esp Geriatr Gerontol. 2002;37 Suppl 2:74105.

2. Liu XZ, Yan D. Aging and hearing loss. J Pathol. 2007;211:188-97.

3. Beers MH, Berkow R. Hearing loss. En: Beers MH, Berkow R, editores. The Merck Manual of Geriatrics. 3.a ed. Whitehouse Station: Merck Research Laboratory; 2000. p. 1317-28.

4. Instituto Nacional de Estadística. Encuesta de discapacidades, deficiencias y estado de salud. Madrid: Instituto Nacional de Estadística; 2008.

5. Gates GA, Mills JH. Presbycusis. Lancet. 2005;366:1111-20.

6. Jerger J, Chmiel R, Wilson N, Luchi R. Hearing impairment in older adults: new concepts. J Am Geriatr Soc. 1995;43:928-35.

7. Millán-Calenti JC. Trastornos de los órganos de los sentidos. En: Millán-Calenti, J.C., editor. Principios de geriatría y gerontología. Madrid: McGraw-Hill Interamericana; 2006. p. 589-95. 
8. Gates GA, Rees TS. Hear Ye? Hear ye! Successful auditory aging. West J Med. 1997;167:247-52.

9. Boettcher FA. Presbyacusis and the auditory brainstem response. J Speech Lang Hear Res. 2002;45:1249-61.

10. Sprinzl GM, Riechelmann H. Current trends in treating hearing loss in elderly people: a review of the technology and treatment options -A mini-review. Gerontology. 2010;56:351-8.

11. Popelka MM, Cruickshanks KJ, Wiley TL, Tweed TS, Klein BE, Klein R. Low prevalence of hearing aid use among older adults with hearing loss: the Epidemiology of Hearing Loss Study. J Am Geriatr Soc. 1998;46:1075-8.

12. Natalizia A, Casale M, Guglielmelli E, Rinaldi V, Bressi F, Salvinelli F. An overview of hearing impairment in older adults: perspectives for rehabilitation with hearing aids. Eur Rev Med Pharmacol Sci. 2010;14:223-9.

13. Park S, Johnson MA. What is an adequate dose of oral vitamin B12 in older people with poor vitamin B12 status? Nutr Rev. 2006;64:373-8.

14. Seidman MD. Effects of dietary restriction and antioxidants on presbyacusis. Laryngoscope. 2000;110:727-38

15. Beekman AT, Copeland JR, Prince MJ. Review of community prevalence of depression in later life. Br J Psychiatry. 1999; 174:307-11.

16. Unützer J, Patrick DL, Simon G, Grembowski D, Walker E, Rutter C, et al. Depressive symptoms and the cost of health services in HMO patients aged 65 and older: a 4-year prospective study. JAMA. 1997;277:1618-23.

17. Hickie I, Scott E, Naismith S, Ward PB, Turner K, Parker G, et al. Lateonset depression: genetic, vascular, and clinical contributions. Psychol Med. 2001;31:1403-12.

18. Jansson M, Gatz M, Berg S, Johansson B, Malmberg B, McClearn GE, et al. Association between depressed mood in the elderly and a 5-HTR2A gene variant. Am J Med Genet B Neuropsychiatr Genet. 2003;120:79-84.

19. Carson A, Margolin R. Depression in older patients with neurologic illness: causes, recognition, management. Cleve Clin J Med. 2005;72 Suppl 3:52-64.

20. Jorm AF. Is depression a risk factor for dementia or cognitive decline? A review. Gerontology. 2000;46:219-27.

21. Alexopoulos GS, Meyers BS, Young RC, Campbell S, Silbersweig D, Charlson M. Vascular depression hypothesis. Arch Gen Psychiatry. 1997;54:915-22.

22. Schrag A. Quality of life and depression in Parkinson's disease. J Neurol Sci. 2006;248:151-7.

23. Chwastiak L, Ehde DM, Gibbons LE, Sullivan M, Bowen JD, Kraft GH. Depressive symptoms and severity of illness in multiple sclerosis: epidemiologic study of a large community sample. Am J Psychiatry. 2002;159:1862-8.

24. Kanner AM, Balabanov A. Depression and epilepsy: how closely related are they? Neurology. 2002;58(8 Suppl 5):27-39.

25. Alexopoulos GS. Clinical and biological findings in late-onset depression. En: Tasman A, Goldfinger SM, Kaufmann CA, editores. American psychiatric press review of psychiatry. Washington: American Psychiatric Press; 1990. p. $249-62$.

26. Millán-Calenti JC, Tubío J, Pita-Fernández S, González-Abraldes I, Lorenzo T, Maseda A. Prevalence of cognitive impairment: effects of level of education, age, sex and associated factors. Dement Geriatr Cogn Disord. 2009;28:455-60.

27. Kalayam B, Meyers BS, Kakuma T, Alexopoulos GS, Young RC, Solomon S, et al. Age at onset of geriatric depression and sensorineural hearing deficits. Biol Psychiatry. 1995;38:649-58.

28. Grace J, O'Brien JT. Association of life events and psychosocial factors with early but not late onset depression in the elderly: implications for possible differences in aetiology. Int J Geriatr Psychiatry. 2003;18:473-8.

29. Alexopoulos GS. Depression in the elderly. Lancet. 2005;365:1961-70.

30. Blazer DG, Moody-Ayers S, Craft-Morgan J, Burchett B. Depression in diabetes and obesity: racial/ethnic/gender issues in older adults. J Psychosom Res. 2002;53:913-6.

31. Magaziner J, Simonsick E, Kashner M. Predictors of functional recovery in the years following hospital discharge for hip fracture. J Gerontol. 1990;45:101-7.

32. Black SA, Goodwin JS, Markides KS. The association between chronic diseases and depressive symptomatology in older Mexican Americans. J Gerontol A Biol Sci Med Sci. 1998;53:188-94.

33. Millán-Calenti JC, Tubío J, Pita-Fernández S, González-Abraldes I, Lorenzo T, Fernández-Arruty T, et al. Prevalence of functional disability in activities of daily living (ADL), instrumental activities of daily living (IADL) and associated factors, as predictors of morbidity and mortality. Arch Gerontol Geriatr. 2009;50:306-10.

34. Blazer II DG, Hybels CF. Origins of depression in later life. Psychol Med. 2005;35:1241-52.

35. Morse JQ, Lynch TR. A preliminary investigation of self-reported personality disorders in late life: prevalence, predictors of depressive severity, and clinical correlates. Aging Ment Health. 2004;8:307-15.

36. Martin P, Long MV, Poon LW. Age changes and differences in personality traits and states of the old and very old. J Gerontol B Psychol Sci Soc Sci. 2002;57:144-52.

37. Lyness JM, Caine ED, King DA, Conwell Y, Duberstein PR, Cox C. Depressive disorders and symptoms in older primary care patients: one-year outcomes. Am J Geriatr Psychiatry. 2002;10:275-82.

38. Steunenberg B, Beekman AT, Deeg DJ, Kerkhof AJ. Personality and the onset of depression in late life. J Affect Disord. 2006;92:243-51.

39. Katon W, Lin E, Von Korff M, Bush T, Walker E, Simon G, et al. The predictors of persistence of depression in primary care. J Affect Disord. 1994;31:81-90.

40. Mazure CM, Maciejewski PK, Jacobs SC, Bruce ML. Stressful life events interacting with cognitive/personality styles to predict late-onset major depression. Am J Geriatr Psychiatry. 2002;10:297-304.

41. Bisschop MI, Kriegsman DM, Beekman AT, Deeg DJ. Chronic diseases and depression: the modifying role of psychosocial resources. Soc Sci Med. 2004;59:721-33.

42. Bruce ML. Psychosocial risk factors for depressive disorders in late life. Biol Psychiatry. 2002;52:175-84.

43. Erber NP, Scherer SC. Sensory loss and communication difficulties in the elderly. Australasian J Ageing. 1999;18:4-9.

44. Dalton DS, Cruickshanks KJ, Klein BE, Klein R, Wiley TL, Nondahl DM. The impact of hearing loss on quality of life in older adults. Gerontologist. 2003;43: 661-8.

45. Hogan A, O'Loughlin K, Miller P, Kendig H. The health impact of a hearing disability on older people in Australia. J Aging Health. 2009;21: 1098-111.

46. Jones DA, Victor CR, Vetter NJ. Hearing difficulty and its psychological implications for the elderly. J Epidemiol Community Health. 1984;38:75-8.

47. Abrams TE, Barnett MJ, Hoth A, Schultz S, Kaboli PJ. The relationship between hearing impairment and depression in older veterans. J Am Geriatr Soc. 2006;54:1475-7.

48. Saito H, Nishiwaki Y, Michikawa T, Kikuchi Y, Mizutari K, Takebayashi T, et al. Hearing handicap predicts the development of depressive symptoms after 3 years in older community-dwelling Japanese. JAGS. 2010;58:93-7.

49. Brink P, Stones M. Examination of the relationship among hearing impairment, linguistic communication, mood, and social engagement of residents in complex continuing-care facilities. Gerontologist. 2007;47:633-41. 
50. Cacciatore F, Napoli C, Abete P, Marciano E, Triassi M, Rengo F. Quality of life determinants and hearing function in an elderly population: Osservatorio Geriatrico Campano Study Group. Gerontology. 1999;45:323-8.

51. Poissant SF, Beaudoin F, Huang J, Brodsky J, Lee DJ. Impact of cochlear implantation on speech understanding, depression, and loneliness in the elderly. J Otolaryngol Head Neck Surg. 2008;37:488-94.

52. Metselaar M, Maat B, Krijnen P, Verschuure H, Dreschler WA, Feenstra L. Selfreported disability and handicap after hearingaid fitting and benefit of hearing aids: comparison of fitting procedures, degree of hearing loss, experience with hearing aids and uni- and bilateral fittings. Eur Arch Otorhinolaryngol. 2009;266:907-17.

53. Cox RM, Alexander GC, Gray GA. Personality, hearing problems, and amplification characteristics: contributions to self-report hearing aid outcomes. Ear Hear. 2007;28:141-62.

54. Oppegard K, Hansson RO, Morgan T, Indart M, Crutcher M, Hampton P. Sensory loss, family support, and adjustment among the elderly. J Soc Psychol. 1984;123:291-2.

55. López-Torres Hidalgo J, Boix Gras C, Téllez Lapeira J, López Verdejo A, DelCampo del Campo JM, Escobar Rabadán F. Functional status of elderly people with hearing loss. Arch Gerontol Geriatr. 2009;49:88-92. 